\title{
Consumo de alcohol y su relación con la agresividad en adolescentes de secundaria
}

\author{
Paola Lizeth Flores-Garza', Karla Selene López-García', Francisco Rafael Guzmán-Facundo', Lucio \\ Rodríguez-Aguilar', Benito Israel Jiménez-Padilla' \\ I Facultad de Enfermería, Subdirección de Posgrado e Investigación, Universidad Autónoma de Nuevo León, Monterrey, México
}

\begin{abstract}
RESUMEN
Introducción: el consumo de alcohol es un problema de salud que impacta negativamente en el bienestar biopsicosocial de las personas y puede favorecer la aparición de distintas formas de agresividad. Objetivo: determinar la relación entre la frecuencia y tipo de consumo de alcohol y distintas formas de agresividad en adolescentes de secundaria. Método: se realizó un estudio descriptivo correlacional en 259 adolescentes de secundaria del estado de Nuevo León, México, a quienes se les aplicó una cédula de datos personales y consumo de alcohol, así como el cuestionario AUDIT y el cuestionario de agresividad (AQ) de Buss y Perry (1992). Resultados: se encontró que 122 adolescentes han consumido alcohol alguna vez en su vida, de los cuales $57.7 \%$ presenta un consumo de riesgo y $28.9 \%$ un consumo dependiente. Asimismo, se observó una media de 40.3 en la escala de agresividad general, se identificó a la ira como el tipo de agresividad que presentó la mayor puntuación $(M=42.0)$. Además, tanto la agresividad general como la agresividad física se relacionaron con el consumo de riesgo $\left(r_{\mathrm{s}}=.213, p=.036\right.$, en el caso de la agresividad general y $r_{\mathrm{s}}=.287, p=.004$ en el de la agresividad física) y con el consumo dependiente $\left(r_{\mathrm{s}}=\right.$ .209, $p=.040$, para la agresividad general y $r_{\mathrm{s}}=.219, p$ $=.031$ para la agresividad física). Conclusiones: tanto quienes presentan un consumo de riesgo como quienes presentan dependencia del alcohol manifiestan mayores niveles de agresividad general y física.
\end{abstract}

Palabras clave: consumo de alcohol, agresividad, adolescentes.

\begin{abstract}
Introduction: alcohol consumption is a health problem that impacts negatively in biopsychosocial well-being of people and may favor the appearance of aggression. Objective: determine alcohol consumption (frequency and type of consumption) and aggressiveness in middle school adolescents. Methods: a correlational descriptive study was conducted in 259 students of high School in Monterrey, Mexico. A personal and alcohol consumption data card was applied as well as the AUDIT questionnaire and the Aggression Questionnaire (AQ) by Buss and Perry (1992). Results: showed that 122 adolescents have consumed alcohol some time in life, of which $57.7 \%$ presented hazardous consumption and $28.9 \%$ dependence consumption. The mean for general aggression scale was 40.3 and the anger was the type of aggression with the higher mean score $(M=42.0)$. Also, it was identified that general aggression correlates with hazardous alcohol consumption $\left(r_{\mathrm{s}}=.213, p=.036\right)$ and dependent consumption ( $\left.r_{\mathrm{s}}=.209, p=.040\right)$; physical aggression were also related to hazardous alcohol consumption $\left(r_{\mathrm{s}}=.287, p=.004\right)$ and dependence consumption $\left(r_{\mathrm{s}}=.219, p=.031\right)$. Conclusion: adolescents with hazardous and dependence alcohol consumption were more likely to present aggressiveness.
\end{abstract}

Keywords: alcohol consumption, aggressiveness, adolescents.

\footnotetext{
Autor de correspondencia:

Paola Lizeth Flores Garza. Facultad de Enfermería, Subdirección de Posgrado e Investigación, Universidad Autónoma de Nuevo León, Monterrey, México. Av. Gonzalitos núm. 1500 Nte., Col Mitras Centro, Monterrey, Nuevo León, México. C.P. 64460, Teléfono 83481847. Correo electrónico: pao_flores46@hotmail.com

Recibido: 27 de agosto de 2018.

Aceptado: 26 de marzo de 2019.

doi: 10.28931/riiad.2019.1.05
} 


\section{INTRODUCCIÓN}

En la actualidad, el consumo de alcohol es un problema de salud pública que impacta negativamente en el bienestar físico, social y mental de las personas, y genera repercusiones en la salud, educación y seguridad pública. Las estadísticas publicadas por la Organización Mundial de la Salud (OMS) en el 2015 reportaron que $32 \%$ de los adolescentes de edades comprendidas entre los 13 y los 15 años consumían alcohol y presentaban un consumo superior al resto de la población. (OMS, 2016b). El Fondo de las Naciones Unidas para la Infancia (UNICEF [por sus siglas en inglés]; 2002) menciona que los adolescentes son más vulnerables al consumo de alcohol, debido a los cambios físicos, cognitivos y conductuales de esta etapa, además de que su cerebro está en pleno desarrollo y tienen mayor riesgo de crear dependencia o abuso a largo plazo, así como sufrir las consecuencias de este consumo, incluso la muerte (OMS, 2016a).

En América Latina, $40 \%$ de las mujeres y $54 \%$ de los hombres entre 12 y 18 años consumieron alcohol en el 2016 (UNODC, 2017). En México, el Sistema Nacional de Vigilancia Epidemiológica de las Adicciones (SISVEA, 2015) reportó que el alcohol es la droga inicial de consumo, con 41.4\%; la Encuesta Nacional de Consumo de Drogas, Alcohol y Tabaco (ENCODAT) 2016-2017 (Villatoro-Velázquez et al., 2017) muestra una tendencia similar en el consumo de alcohol en la población adolescente, ya que la prevalencia alguna vez en la vida pasó de $35.6 \%$ en 2002 a $42.9 \%$ en 2011; esta prevalencia se mantuvo en 2017 , que fue de $39.8 \%$ (47\% hombres y $37.9 \%$ mujeres). El consumo en el último año pasó de $25.7 \%$ en 2002 a $30 \%$ en 2011 , y es similar a lo reportado en 2017 , que fue de $28 \%$ (28.8\% hombres y $27.2 \%$ mujeres). Asimismo, $8.3 \%$ de la población adolescente (8.9\% de los hombres y $7.7 \%$ de las mujeres) presenta un consumo excesivo de alcohol y $0.8 \%$ es dependiente de esta sustancia.

Debido a la problemática del consumo por parte de los adolescentes, la OMS (2016b) establece que el consumo de alcohol tiene consecuencias perjudiciales en la salud, como embarazos no deseados, enfermedades infecciosas, de transmisión sexual y desajuste en las rutinas de sueño. La inmadurez cerebral, la falta del control de las emociones e impulsos y el descontrol hormonal en los adolescentes pueden provocar cambios negativos en su conducta, como la agresividad, manifestada a través de la agresión física y verbal, ira y hostilidad, las cuales tienen consecuencias negativas en la salud y calidad de vida de los adolescentes, así como muerte prematura (Moñino, Piñero, Árense, \& Cerezo, 2013; UNICEF, 2002).
De acuerdo con la literatura, $79.9 \%$ de los adolescentes ha experimentado agresividad y de estos, $10 \%$ refiere haber agredido a alguien bajo los efectos del alcohol. Este binomio es una de las principales causas de mortalidad, ya que diariamente fallecen 180 adolescentes relacionados con la agresividad física, además de causar lesiones y discapacidades que perduran toda la vida y que afectan su funcionamiento fisiológico, psicológico y social (Chavarriaga-Rios \& Segura-Cardona, 2015; De Almeida et al., 2014).

Este binomio ha sido estudiado en diferentes países como España, Chile, Colombia y México, donde tuvo una importante relevancia debido a la escasa evidencia que existe de ambas variables y a la problemática que representa el consumo de alcohol y la agresividad en la población adolescente, aunado al problema que enfrenta el país por las conductas agresivas relacionadas con el narcotráfico y los diferentes contenidos en los medios de comunicación. Por ello, es de vital importancia trabajar desde la perspectiva de enfermería, con la finalidad de conocer más acerca del fenómeno de estudio en el país y disponer de mejores elementos para implementar intervenciones enfocadas en la promoción de la salud, la prevención y la detección del consumo problemático de alcohol, así como la reinserción social de los adolescentes que lo presentan, además de la prevención, detección y atención de la agresividad en esta población. Por lo anterior, el presente estudio tiene como objetivo determinar la prevalencia y el tipo de consumo de alcohol y su relación con la agresividad general y por sexo en los adolescentes de secundaria.

\section{MÉTODO}

El diseño del estudio fue descriptivo y correlacional (Burns \& Grove, 2012), ya que se describió la prevalencia del consumo de alcohol y la agresividad en adolescentes y la relación entre estas dos variables en los adolescentes de ambos sexos de dos secundarias públicas.

\section{Muestra}

Se seleccionaron por conveniencia dos escuelas secundarias públicas con una población total de 1,200 estudiantes de ambos sexos, el muestreo fue de tipo estratificado con asignación proporcional al tamaño del estrato (Secundaria A y Secundaria B) y dentro de cada estrato se realizó un muestreo por conglomerados (grupos). El tamaño de muestra se determinó con base en una correlación, con un nivel de significancia de .05 para una hipótesis bilateral alternativa, se consideró una potencia de $90 \%$, un efecto de diseño de 1.15 y se obtuvo una muestra de 259 estudiantes. 


\section{Instrumento}

Para el presente estudio se utilizó una cédula de datos personales conformada por tres preguntas: edad, género y grado académico. Para medir el consumo de alcohol que se refiere a la ingesta por parte de los adolescentes de secundaria se utilizaron prevalencias (global, lápsica, actual e instantánea). Para conocer el tipo de consumo de alcohol se utilizó el Cuestionario de Identificación de los Trastornos debido al consumo de AIcohol (AUDIT, por sus siglas en inglés) por Babor, Higgins-Biddle, Saunders y Monteiro (2001). El cuestionario consta de 10 ítems, que miden el consumo de riesgo, el consumo dependiente y el consumo dañino. Para el procesamiento estadístico se realizaron sumatorias de los puntajes, a fin de obtener el tipo de consumo de alcohol. En el presente estudio, el instrumento presentó un Alpha de .78.

La agresividad, definida como la percepción que tiene el adolescente sobre su comportamiento derivado de pensamientos hostiles y/o sentimientos negativos que lo pueden llevar a dañar físicamente o herir verbalmente a otra persona, se midió a través del cuestionario de agresividad elaborado por Buss y Perry (1992), que está conformado por 29 ítems relacionados con conductas y sentimientos agresivos, los cuales están codificados en una escala de tipo Likert de cinco puntos. Se estructura en cuatro dimensiones, distribuidas en las escalas de agresividad verbal y física, de hostilidad y de ira. Para la interpretación del cuestionario, de acuerdo con las normas de calificación y corrección, a cada ítem se le otorga el puntaje según el número marcado, exceptuando los dos ítems negativos (15 y 24) donde su puntaje se invierte. Posteriormente, se realiza la sumatoria de los puntajes por dimensiones, en la que se puede concluir que a mayor puntaje, mayor es la agresividad que se presenta en el adolescente. La confiabilidad de este instrumento fue aceptable $(a=.91)$.

\section{Procedimiento}

Una vez obtenido el dictamen favorable de los Comités de Ética en Investigación e Investigación de una universidad pública del país y de las instituciones educativas seleccionadas, se visitó cada una de ellas tras solicitar la autorización del maestro presente en el aula para invitar a los estudiantes a participar en la práctica. La visita a los grupos de alumnos se realizó en horarios indicados por las autoridades de la escuela secundaria, de tal manera que se cuidó no interferir con la carga académica programada de los estudiantes.

Una vez frente al grupo, el investigador principal explicó de forma clara y sencilla las características y obje- tivos del estudio y entregó las cartas de consentimiento informado para la firma de los padres o tutores del adolescente. Se acudió para la recolección de estas cartas y se entregó la carta de asentimiento informado a los adolescentes que aceptaron participar en la aplicación de los instrumentos, en el siguiente orden: la cédula de datos personales y de prevalencia de consumo de alcohol, el Cuestionario de Identificación de los Trastornos debido al consumo de Alcohol (AUDIT) y el Cuestionario de Agresividad. Se explicó a los participantes, en el mismo orden, la forma adecuada de dar respuesta a cada uno estos y se hizo hincapié en la confidencialidad y el anonimato de los datos.

\section{Análisis estadístico}

Para el análisis de los datos se utilizó estadística descriptiva e inferencial. El análisis descriptivo se realizó a través de frecuencias, proporciones, medidas de tendencia central y variabilidad. Para determinar la normalidad en la distribución de las variables continuas, se realizó la Prueba de Bondad de Ajuste de Kolmogorov-Smirnov con corrección de Lilliefors, donde se concluyó que las variables no presentaron distribución normal, por lo cual se decidió utilizar estadística No Paramétrica. Dentro de la estadística Inferencial se aplicaron las pruebas de Chi cuadrada de Pearson, Prueba $U$ de Mann-Whitney y el Coeficiente de Correlación de Spearman. Todos los datos fueron procesados mediante el paquete estadístico Statistical Package for the Social Sciences (SPSS) versión 21.0.

\section{Consideraciones éticas}

El presente estudio fue aprobado por los Comités de Ética en Investigación e Investigación de una universidad pública del país y se apegó a lo establecido en el Reglamento de la Ley General de Salud en Materia de Investigación para la Salud (SSA, 1987), la cual establece que para el desarrollo de investigación en salud se deben considerar aspectos éticos que garanticen la dignidad y el bienestar de los participantes en la investigación. En este sentido, por ser adolescentes menores de edad, se elaboraron cartas de consentimiento informado para el padre o tutor, a fin de autorizar la participación del adolescente en el presente estudio. Asimismo, se elaboró una carta de asentimiento informado para que el alumno expresara su aceptación a participar en el estudio y en la que se le indicaba que si lo deseaba podía suspender su participación en el momento que lo decidiera sin que eso repercutiera en su rol como estudiante o como integrante de la sociedad. 


\section{RESULTADOS}

Dentro de los resultados encontrados se observó que los adolescentes presentaron una media de 13.5 años, se mostraron proporciones similares de estudiantes del sexo masculino (47.5\%) y del femenino (52.5\%). Respecto al grado escolar, se reporta que los de tercer año son los que presentaron mayor proporción (40.5\%). En cuanto a las frecuencias del consumo de alcohol, se pudo observar que 122 adolescentes (47.1\%) han consumido alcohol alguna vez en la vida, 97 (37.5\%) lo consumieron en el último año y 50 adolescentes (19.3\%) en los últimos 30 días; sólo 20 (7.2\%) lo consumieron en los últimos siete días (Tabla 1). La edad de inicio del consumo de esta droga es en promedio a los 12.5 años; con respecto al patrón de consumo se observó que de los adolescentes que han bebido alcohol, $57.7 \%$ presenta un consumo de riesgo, $28.9 \%$ un consumo dependiente y $13.4 \%$ un consumo perjudicial (Tabla 2).

En cuanto a las diferencias del uso de alcohol por sexo, se encontró que son las mujeres quienes registran el mayor en las cuatro prevalencias (global 50.0\%; lápsica 41.2\%; actual 25.7\%; e instantánea 12.5\%). Sin embargo, sólo en las prevalencias actual e instantánea se presentaron diferencias estadísticamente significativas $\left(x^{2}=7.6\right.$ y $X^{2}=9.1, p<.05$ respectivamente); se indica que las mujeres presentan un consumo mayor

Tabla 1

Prevalencias del consumo de alcohol en adolescentes

\begin{tabular}{lrrrrr}
\hline \multicolumn{1}{c}{ Variable } & \multicolumn{2}{c}{ Sí } & \multicolumn{3}{c}{ NO } \\
& \multicolumn{1}{c}{$f$} & $\%$ & \multicolumn{1}{c}{$f$} & $\%$ \\
\hline Consumo de alcohol & & & & \\
Alguna vez en la vida & 122 & 47.1 & 137 & 52.9 \\
En el último año & 97 & 37.5 & 162 & 62.5 \\
En los últimos 30 días & 50 & 19.3 & 209 & 80.7 \\
En los últimos 7 días & 20 & 7.2 & 239 & 92.3 \\
\hline Nota: $f=$ frecuencias, \% = porcentaje & \multicolumn{4}{c}{$(n=259)$}
\end{tabular}

que los hombres en el último mes y en los últimos siete días (Tabla 3).

En lo que respecta a la agresividad en los adolescentes, se encontró que presentaron una media de 40.3 en la escala de agresividad general. La ira fue el tipo de agresividad más frecuente en los adolescentes participantes $(M=42.0)$, seguida por la hostilidad $(M=41.9)$, posteriormente la agresividad verbal $(M=37.9)$ y por último la agresividad física $(M=31.6)$.

En relación con las diferencias por sexo, la agresividad en general en el sexo femenino $(M=41.2)$ presenta una media mayor con relación al sexo masculino $(M=$ 39.1). En cuanto a los tipos de agresividad (Tabla 4), en la física los hombres presentaron niveles más altos $(M=34.1)$, comparados con las mujeres $(M=29.4)$, mostrando una diferencia estadísticamente significativa $(p<.05)$. Respecto a la agresividad verbal, la media más alta se presentó en el sexo femenino $(M=38.4)$ y en el tipo de agresividad de ira y hostilidad las mujeres presentaron las medias más altas $(M=45.9, M=44.3$, respectivamente), lo que indica una diferencia significativa para estos dos tipos de agresividad $(p<.05)$.

También sé identificó que la agresividad se relacionó positiva y significativamente con el consumo de riesgo de alcohol $\left(r_{\mathrm{s}}=.213, p=.036\right)$ y con el consumo dependiente $\left(r_{\mathrm{s}}=.209, p=.040\right)$, lo que significa que los estudiantes que tienen un consumo de riesgo y dependiente de alcohol presentan mayor agresividad. En cuanto al tipo de agresividad, la física se relacionó positiva y significativamente con el consumo de riesgo $\left(r_{\mathrm{s}}=.287, p=.004\right)$ y dependiente de alcohol $\left(r_{\mathrm{s}}=.219\right.$, $p=.031$ ), lo cual quiere decir que los adolescentes con consumo de riesgo y consumo dependiente de alcohol presentan mayor agresividad física (Tabla 5).

\section{DISCUSIÓN Y CONCLUSIONES}

Identificar el consumo de alcohol en los adolescentes y su relación con la agresividad (física, verbal, ira y hostilidad) es de suma importancia debido a las conse-

Tabla 2

Tipo de consumo de alcohol en los adolescentes

\begin{tabular}{lccccc}
\hline & $\begin{array}{c}\text { Tipo de consumo } \\
(n=97)\end{array}$ & $f$ & $\%$ & \multicolumn{2}{c}{ IC 95\% } \\
\cline { 4 - 6 } & & 56 & 57.7 & 48.0 & 68.0 \\
De riesgo & 28 & 28.9 & 19.0 & 37.0 \\
Dependiente & 13 & 13.4 & 6.0 & 20.0 \\
Perjudicial & & 13 & LI & LS \\
\hline
\end{tabular}

Nota: $n=$ total de observaciones; $f=$ frecuencias; $\%=$ porcentaje; $I C=$ Intervalo de confianza; $L I=$ límite inferior;

LS = límite superior 
Tabla 3

Prevalencia de consumo de alcohol en los adolescentes por sexo

\begin{tabular}{|c|c|c|c|c|c|c|c|c|c|c|}
\hline \multirow{3}{*}{ Variable } & \multicolumn{4}{|c|}{ Masculino } & \multicolumn{4}{|c|}{ Femenino } & \multirow{3}{*}{$x^{2}$} & \multirow{3}{*}{$p$} \\
\hline & \multicolumn{2}{|c|}{ Sí } & \multicolumn{2}{|c|}{ NO } & \multicolumn{2}{|c|}{ Sí } & \multicolumn{2}{|c|}{ NO } & & \\
\hline & $f$ & $\%$ & $f$ & $\%$ & $f$ & $\%$ & $f$ & $\%$ & & \\
\hline \multicolumn{11}{|l|}{ Alcohol } \\
\hline Global & 54 & 43.9 & 69 & 56.1 & 68 & 50.0 & 68 & 50.0 & 0.9 & .326 \\
\hline Lápsica & 41 & 33.3 & 82 & 66.7 & 56 & 41.2 & 80 & 58.8 & 1.6 & .193 \\
\hline Actual & 15 & 12.2 & 108 & 87.8 & 35 & 25.7 & 101 & 74.3 & 7.6 & .006 \\
\hline Instantánea & 3 & 2.4 & 120 & 97.6 & 17 & 12.5 & 119 & 87.5 & 9.1 & .002 \\
\hline
\end{tabular}

Tabla 4

Agresividad física, verbal, ira y hostilidad en los adolescentes por sexo

\begin{tabular}{|c|c|c|c|c|c|}
\hline $\begin{array}{l}\text { Variables } \\
(n=259)\end{array}$ & $M$ & $D E$ & Mdn & $U$ & $p$ \\
\hline \multicolumn{6}{|l|}{ Agresividad } \\
\hline Masculino & 39.3 & 17.7 & 38.7 & \multirow{2}{*}{7707.5} & \multirow{2}{*}{.275} \\
\hline Femenino & 41.2 & 18.8 & 43.5 & & \\
\hline \multicolumn{6}{|l|}{ Agresividad física } \\
\hline Masculino & 34.1 & 18.3 & 33.3 & \multirow{2}{*}{7146.0} & \multirow{2}{*}{.043} \\
\hline Femenino & 29.4 & 19.1 & 25.0 & & \\
\hline \multicolumn{6}{|l|}{ Agresividad verbal } \\
\hline Masculino & 37.3 & 22.3 & 35.0 & \multirow{2}{*}{8176.0} & \multirow{2}{*}{.754} \\
\hline Femenino & 38.4 & 24.9 & 40.0 & & \\
\hline \multicolumn{6}{|l|}{ Ira } \\
\hline Masculino & 37.6 & 20.9 & 35.7 & \multirow{2}{*}{6498.0} & \multirow{2}{*}{.002} \\
\hline Femenino & 45.9 & 22.6 & 46.4 & & \\
\hline \multicolumn{6}{|l|}{ Hostilidad } \\
\hline Masculino & 39.3 & 19.8 & 40.6 & \multirow{2}{*}{7185.0} & \multirow{2}{*}{.050} \\
\hline Femenino & 44.3 & 21.3 & 46.8 & & \\
\hline
\end{tabular}

Nota: $n=$ total de observaciones; $M=$ media; $D E=$ Desviación Estándar; $M d n=$ Mediana; $U=U$ de Mann Whitney; Valor de $p=$ significancia

Tabla 5

Coeficiente de Correlación de Spearman para tipo de consumo de alcohol y agresividad en los adolescentes

\begin{tabular}{|c|c|c|c|c|c|}
\hline Variables & $\begin{array}{c}\text { Índice } \\
\text { Agresividad } \\
(\mathrm{AQ})\end{array}$ & $\begin{array}{l}\text { Agresividad } \\
\text { física }\end{array}$ & $\begin{array}{c}\text { Agresividad } \\
\text { verbal }\end{array}$ & Ira & Hostilidad \\
\hline Consumo de alcohol (AUDIT) & $\begin{array}{c}.180 \\
(.077)\end{array}$ & $\begin{array}{l}.194 \\
(.057)\end{array}$ & $\begin{array}{c}.089 \\
(.387)\end{array}$ & $\begin{array}{c}.158 \\
(.121)\end{array}$ & $\begin{array}{l}.146 \\
(.153)\end{array}$ \\
\hline Consumo de riesgo de alcohol & $\begin{array}{l}.213^{\star} \\
(.036)\end{array}$ & $\begin{array}{l}.287^{\star} \\
(.004)\end{array}$ & $\begin{array}{l}.114 \\
(.264)\end{array}$ & $\begin{array}{l}.146 \\
(.152)\end{array}$ & $\begin{array}{l}.120 \\
(.244)\end{array}$ \\
\hline Consumo dependiente de alcohol & $\begin{array}{l}.209^{\star} \\
(.040)\end{array}$ & $\begin{array}{l}.219^{\star} \\
(.031)\end{array}$ & $\begin{array}{l}.045 \\
(.665)\end{array}$ & $\begin{array}{c}.159 \\
(.239)\end{array}$ & $\begin{array}{c}.171 \\
(.094)\end{array}$ \\
\hline Consumo perjudicial de alcohol & $\begin{array}{c}.110 \\
(.283)\end{array}$ & $\begin{array}{c}.097 \\
(.344)\end{array}$ & $\begin{array}{l}.118 \\
(.250)\end{array}$ & $\begin{array}{c}.121 \\
(.239)\end{array}$ & $\begin{array}{c}.079 \\
(.439)\end{array}$ \\
\hline
\end{tabular}

Nota: ${ }^{* \star} p<.001 .{ }^{*} p<.05$ 
cuencias que este binomio les ocasionan. El consumo de alcohol reduce el autocontrol, aumenta las conductas antisociales, como la agresividad, y puede llegar a convertirse en violencia, responsable de la muerte prematura de 180 adolescentes al día, lo que la convierte en una de las principales causas de mortalidad en esta población, además de lesiones y discapacidades que pueden tener repercusiones graves, debido a que muchas de ellas perduran toda la vida y afectan el funcionamiento fisiológico, psicológico y social del adolescente (OMS, 2016c).

En el presente estudio se observó que el consumo de alcohol de los adolescentes es mayor a lo reportado en la ENCODAT 2016-2017 (Villatoro-Velázquez et al., 2017) y que más de la mitad de los adolescentes presenta consumo de riesgo, similar a lo reportado en estudios realizados por Alonso-Castillo, Yáñez-Lozano y Armendáriz-García (2017), Gardea, López, Alonso, Alonso y Alonso (2015) y López-Cisneros, Alonso Castillo, Méndez Ruíz y Armendáriz García (2016), lo cual podría explicarse por la desmedida aceptación social y la baja percepción de riesgo que tienen los adolescentes acerca de las consecuencias de beber alcohol, ya que esta droga está presente en la mayoría de los eventos sociales, deportivos y familiares a los que asisten, probablemente por la alta permisividad de los padres, el hecho de desear un sentimiento de libertad y desinhibición al consumirlo y querer pertenecer a un grupo social. Además, otro factor importante son las redes sociales y los medios publicitarios, que podrían influir de manera negativa en los adolescentes, al incrementar las actitudes y creencias positivas hacia el consumo de esta sustancia.

También se identificó que el sexo femenino es el que tiene mayor consumo de alcohol, lo que difiere de lo reportado por otros autores (De Almeida et al., 2014; OMS, 2016b; Villatoro-Velázquez et al., 2017), quienes indican que el sexo masculino es el que tiene mayores prevalencias de consumo de alcohol y conductas agresivas. Lo anterior puede ser explicado debido a que en los últimos años el rol de las mujeres en la sociedad ha cambiado. Se puede observar que anteriormente el consumo de alcohol sólo era aceptado en los hombres y ahora es indiferente del sexo que lo consume; esto está ante la vista de las adolescentes, lo que favorece su consumo. Además de querer pertenecer al grupo más popular, aumenta la persuasión que existe en las redes sociales hacia el consumo de alcohol.

Con respecto a la agresividad, se pudo observar que los adolescentes presentan niveles altos de agresividad similar a lo reportado por Chavarriaga-Ríos y Segura-Cardona (2015); la ira presentó mayor prevalencia en ambos sexos, resultado que difiere en diversos estudios donde se reporta que la agresividad física y verbal son los de mayor frecuencia (Castaño-Pérez, Restrepo-Escobar, \& Uribe-Aramburo, 2014; Cleberson-de Souza \& Baccarat-de Godoy, 2016; De Almeida et al., 2014; Gutiérrez-Quintanilla \& Portillo-García, 2016). Los niveles altos de agresividad podrían explicarse debido a las influencias de modelos familiares y sociales; los medios de comunicación o los modelos simbólicos transmitidos gráfica o verbalmente pueden mostrar conductas agresivas como la ira, dándole un valor positivo y convirtiéndolos en los responsables de que la agresión se moldee y propague. La experiencia directa actúa de manera que el adolescente es atacado agresivamente y éste responde de la misma manera.

Se encontraron diferencias significativas respecto a la agresividad por sexo. Las mujeres mostraron mayores niveles de ira y hostilidad, similar a lo reportado por De Almeida et al. (2014), mientras que los hombres reportaron mayor agresividad física. Estas diferencias pueden explicarse de acuerdo con lo que señala UNICEF (2002), respecto a que la hormona testosterona está relacionada con la agresividad en el sexo masculino; sin embargo, existen otras actividades y funciones cerebrales que son llevadas a cabo por los corticoesteroides y las glándulas pituitaria y adrenocortical, que se han relacionado también con la agresividad y que indican que ésta no posee un carácter sexual específico.

Además, se encontró que el consumo de riesgo y dependiente de alcohol se relacionaron positiva y significativamente con la agresividad y la subescala de agresividad física en los adolescentes. Lo que significa que a mayor consumo de alcohol, mayor será la agresividad que presenta el adolescente, exponiéndolo a las consecuencias negativas tanto del consumo como de la agresividad. Estos resultados coinciden con los estudios de otros autores que han sido realizados en población adolescente de diferentes países (Castaño-Pérez et al., 2014; Chavarriaga-Rios \& Segura-Cardona, 2015; Cleberson-de Souza \& Baccarat-de Godoy, 2016; Gardea et al., 2015; Gazquez et al., 2016).

Esto pudiera explicarse debido a los cambios neurológicos que produce el alcohol en el cerebro del adolescente, ya que puede inhibir ciertas funciones normales e impedir que éste tenga el control de sus emociones y comportamientos. Aunado a esto, los factores del contexto social, escolar o familiar pueden explicar la relación del porqué se presenta este consumo y las conductas de agresividad en los adolescentes, ya que si en alguno de estos contextos se dan ambos fenómenos, el adolescente puede aprenderlos.

Estos hallazgos son de gran utilidad, ya que permiten observar el comportamiento de estas variables en la población adolescente; además, proporcionan elementos para el desarrollo e implementación de in- 
tervenciones enfocadas en la prevención y la reducción del consumo de alcohol, en las que se consideren las consecuencias de éste, entre ellas la agresividad y la afectación del desarrollo fisiológico, psicológico y social.

En conclusión, se puede observar que el consumo de alcohol está relacionado con la agresividad en los adolescentes y que a mayor consumo, mayor presencia de conductas agresivas. Asimismo, los adolescentes que presentan altas prevalencias de consumo de alcohol e inician el uso de esta sustancia a edades tempranas tienen mayor riesgo de desarrollar dependencia en el futuro, aunado al hallazgo de que las mujeres son quienes consumen mayor cantidad de alcohol y presentan mayor agresividad.

Como recomendaciones, se sugiere seguir utilizando el cuestionario de agresividad de Buss y Perry (1992) en población mexicana, debido a la consistencia interna aceptable que presentó en este estudio, así como realizar investigaciones con una muestra mayor de secundarias públicas y privadas para poder hacer generalizaciones acerca de la agresividad durante esta etapa de la vida. Asimismo, realizar estudios bajo el enfoque cualitativo para conocer las percepciones y los significados que los adolescentes atribuyen al consumo de alcohol y practicar estudios de diseño longitudinal.

\section{FUENTES DE FINANCIAMIENTO}

La presente investigación no fue financiada por ningún organismo ni institución.

\section{CONFLICTOS DE INTERÉS}

Los autores manifiestan que no existe ningún conflicto de interés en la realización del manuscrito.

\section{REFERENCIAS}

Alonso-Castillo, M. M., Yáñez-Lozano, Á., \& Armendáriz-García, N. A. (2017). Funcionalidad familiar y consumo de alcohol en adolescentes de secundaria. Revista Salud y drogas, 17(1), 87-96.

Babor, T., Higgins-Biddle, J., Saunders, J., \& Monteiro, M. (2001). Cuestionario de Identificación de trastornos debido al consumo de alcohol (AUDIT). Ginebra, Suiza: Organización Mundial de la Salud.

Burns, N., \& Grove, S. K. (2012). Investigación en Enfermería. 3a ed. España: Elsevier.

Buss, A. H., \& Perry, M. (1992). The aggression questionnaire. Journal of Personality and Social Psychology, 63(3), 452-459. doi: 10.1037/0022-3514.63.3.452

Castaño-Pérez, G. A., Restrepo-Escobar, S. M., \& Uribe-Aramburo, N. (2014). Agresividad, consumo de drogas y "barras bravas" en el futbol. Revista Virtual de la Universidad Católica del Norte, Colombia, 41(1), 79-95

Chavarriaga-Rios, M. C., \& Segura-Cardona, A. M. (2015). Consumo de sustancias psicoactivas y comportamientos violentos en estudiantes de 11-18 años Revista Salud Publica, 17(5), 655-666. doi: 10.15446/rsap.v17n5.3136

Cleberson-de Souza, F., \& Baccarat-de Godoy, M. C. (2016). Violencia escolar entre adolescentes: condiciones de vulnerabilidad. Enfermería Global, 42, 157-170.

De Almeida, R. M. M., Trentini, L. B., Klein, L. A., Macuglia, G. R., Hammer, C., \& Tesmmer, M. (2014). Uso de álcool, drogas, níveis de impulsividade e agressividade em adolescentes do Rio Grande do Sul. Psico, 45(1), 65-72.

Fondo de las Naciones Unidas para la Infancia. (2002). Adolescencia, una etapa fundamental. New York: UNICEF.

Gardea, D., López, K. S., Alonso, B. A., Alonso, M. T. J., \& Alonso, M. M. (2015). Violencia escolar y consumo de alcohol en adolescentes en etapa de secundaria. Revista de Enfermería Herediana, 8(2), 75-81. doi: 10.20453/renh.v8i2.2685

Gazquez, J. J., Pérez, M. C., Molero, M. M., Barragán, A. B., Martos, A. \& Sánchez, C. (2016). Drug use in adolescents in relation to social support and reactive and proactive aggressive behavior. Psicothema, 28(3), 318-322. doi: 10.7334/psicothema 2015.327

Gutiérrez-Quintanilla, J. R., \& Portillo-García, C. B. (2016). Personalidad y conductas agresivas en jóvenes salvadoreños. Entorno, 62, 7-18.

López-Cisneros, M. A., Alonso Castillo, M. M., Méndez Ruíz, M. D. \& Armendáriz García, N. A. (2016). Descripción del consumo de tabaco y alcohol en adolescentes de complementos urbanos del estado de Nuevo León, México. Salud y drogas, 16(2), 127-134.

Matalinares, C. M., Yaringaño, L. J., Uceda, E. J., Fernández, A. E., Huari T., Y., Campos, G. A., \& Villavicencio, C. N. (2012). Estudio Psicométrico de la versión española del cuestionario de agresión de Buss y Perry. Revista de Investigación en Psicología 15(1), 147-161. doi: 10.15381/rinvp.v15i1.3674

Moñino, M., Piñero, E., Árense, J. \& Cerezo, F. (2013). Violencia escolar y consumo de alcohol y tabaco en estudiantes de educación secundaria. European Journal of Investigation in Health Psychology and Education, 3(2), 137-147. doi: 10.30552/ejihpe. v3i2.45

Moore, S. E., Norman, R. E., Sly, P. D., Whitehouse, A. J. O., Zubrick, S. R. \& Scott, J. (2014). Adolescent peer aggression and its association whit mental health and substance use in an Australian cohort. Journal of Adolescence, 37(1), 11-21. doi: 10.1016/j. adolescence.2013.10.006.

Organización Mundial de la Salud [OMS]. (2016a). Adolescentes: riesgos para la salud y soluciones. Recuperado de: http://www. who.int/mediacentre/factsheets/fs345/es/

Organización Mundial de la Salud [OMS]. (2016b). Consumo de alcohol en Adolescentes Recuperado de: http://www.who.int/mediacentre/factsheets/fs349/es/ 
Organización Mundial de la Salud [OMS]. (2016c). Violencia Juvenil. Recuperado de: http://www.who.int/mediacentre/factsheets/ fs356/es/

Secretaria de Salud [SSA]. (1987). Reglamento de la Ley General de Salud en materia de investigación para la salud. Recuperado de: http://www.salud.gob.mx/unidades/cdi/nom/compi/rlgsmis.html

Sistema Nacional de Vigilancia Epidemiológica de las Adicciones [SISVEA]. (2015). Informe Nacional de Adicciones. Recuperado de: http://www.epidemiologia.salud.gob.mx/doctos/infoepid/ inf_sisvea/informes_sisvea_2015.pdf

United Nations Office on Drugs and Crime [UNODC]. (2017). World Drug Report. Recuperado de https://www.unodc.org/documents /wdr2015/World_Drug_Report_2015.pdf
Villatoro-Velázquez, J. A., Mendoza-Meléndez, M. A., Moreno-López, M., Oliva-Robles, N., Fregoso-lto, D., Diana, Bustos-Gamiño, M.,... Medina-Mora, M. E. (2014). Tendencias del uso de drogas en la ciudad de México: Encuesta de estudiantes, octubre 2012. Salud mental, 37(5), 423-435. doi: 10.17711/SM.01853325.2014.050

Villatoro-Velázquez, J. A., Reséndiz-Escobar, E., Mujica Salazar, A., Bretón-Cirett, M., Cañas-Martínez, V., Soto-Hernández, I.,... Mendoza-Alvarado, L. (2017). Encuesta Nacional de Consumo de Drogas, Alcohol y Tabaco 2016-2017: Reporte de Alcohol. Ciudad de México, México: Instituto Nacional de Psiquiatría Ramón de la Fuente Muñiz. 